\title{
Facial Pyoderma Gangrenosum in Senescence
}

\author{
Dorothea Kratzsch Mirjana Ziemer Linda Milkova Justinus A. Wagner \\ Jan C. Simon Michael Kendler
}

Department of Dermatology, Venerology and Allergology, Leipzig University Hospital, Leipzig, Germany

\section{Key Words}

Pyoderma gangrenosum $\cdot$ Senescence $\cdot$ Face $\cdot$ Ulcer

\begin{abstract}
Clinically, pyoderma gangrenosum (PG) is characterized by a rapidly progressive, painful cutaneous ulcer with an irregular, violaceous and undermined border. PG occurs most frequently on the lower extremities and the trunk of middle-aged individuals. The face is only very rarely affected. We present an 89 - and a 90 -year-old patient, who developed a facial ulcer consistent with PG.

(c) 2013 S. Karger AG, Basel
\end{abstract}

\section{Introduction}

Clinically, pyoderma gangrenosum (PG) is characterized by a rapidly progressive, painful cutaneous ulcer with an irregular, violaceous and undermined border. According to the current literature, it is thought to be an inflammatory dermatosis as part of the spectrum of neutrophilic dermatoses [1]. Diagnostic criteria were proposed by $\mathrm{Su}$ et al. [1]. The characteristic clinical appearance together with the exclusion of other causes of cutaneous ulceration was defined as major criteria. Minor criteria include (i) a history suggestive of pathergy, (ii) a systemic disease associated with PG, (iii) histopathologic findings consistent with PG, and (iiii) a rapid response to systemic steroid treatment. Diagnosis requires both, 2 major and at least 2 minor criteria. PG develops most frequently on the lower extremities and the trunk [2], and only rarely in the face. The age at onset of the disease was reported to be $51.6 \pm 15.0$ years (mean \pm SD) [2]. Women are affected more often than men. Less evidence exists for the appearance of PG in senescent patients. We hereby report about facial ulcers consistent with PG in 2 geriatric patients. 


\section{Case 1}

A 90-year-old woman was referred to our department with a 3-week history of an ulcer on the lateral corner of the left eye. The painless wound appeared spontaneously, without any apparent trauma, and it had gradually enlarged. Concerning comorbidities, the patient suffered from hypertension and rheumatoid arthritis. The latter was treated with methotrexate $10 \mathrm{mg} /$ week and prednisolone $2 \mathrm{mg} /$ day. Initial physical examination in our outpatient department showed a deep ulcer on the lateral corner of the left eye, $1.5 \times 1.5 \mathrm{~cm}$ in size, with a necrotic and fibrinoid surface (fig. 1a). The left eyelids were erythematously swollen and showed accompanying conjunctivitis. Histology from a skin biopsy taken from the edge of the ulcer showed a dense, mixed-cell, dermal infiltrate with numerous neutrophilic granulocytes and some macrophages (fig. 1b). Periodic acid-Schiff and Giemsa stains failed to indicate any infectious disease with bacteria or fungi. The laboratory investigation of the peripheral blood revealed normocytic anemia, lymphocytopenia and neutrophilia. Creactive protein was increased with $21.08 \mathrm{mg} / \mathrm{l}$ (normal value $<5 \mathrm{mg} / \mathrm{l}$ ) and antinuclear antibodies were negative. Bacteriological examination of an ulcer smear revealed growth of physiologic skin flora only. To sum up, the lesion was interpreted as PG. We started systemic therapy with high-dose prednisolone $(75 \mathrm{mg} /$ day), gradually tapered after 7 days. For topical treatment, rinsing with a physiological salt solution and ofloxacine eyedrops (5 times/day) were recommended after the ophthalmologic consultation. Within 2 weeks, the ulcer was reduced in size, showed a clean base without necrotic debris, and the border was less inflamed (fig. 1c).

\section{Case 2}

An 89-year-old man was admitted to our department with a post-surgery wound dehiscence on the left part of the lower lip after squamous cell carcinoma surgery 13 days ago (tumor thickness $6.5 \mathrm{~mm}$, depth of invasion level V). A wound revision was performed with suturing of the lesion by a three-layer wound closure and an antibiotic therapy with cefuroxim $500 \mathrm{mg}$ twice daily for 8 days. In the following 6 weeks, the patient was readmitted to our department several times because he suffered from recurrent wound dehiscence. The patient reported that the dehiscence was triggered by mechanical irritation, such as food intake or sneezing. He described a progressive pain in the suture area. Physical examination showed an ulcer with violaceous undermined borders on the left part of the lower lip (fig. 2a). After each admittance, he was treated with an extensive excision of fibrinoid debris and necrotic tissue, following an antiseptic lavage with betaisodona ${ }^{\circledR}$. A synthetic wound dressing (Syspur Derm ${ }^{\circledR}$, Paul Hartmann AG, Germany) was inserted. Bacterial swab examinations were sterile. Furthermore, incisional biopsy from the edge of the wound was performed. Histopathological examination showed a dense neutrophilic dermatitis without any evidence of residual neoplastic disease. Based on clinical and histopathological correlation, PG was suspected. To exclude the possibility of an allergic reaction to suture material, we performed an intracutaneous insertion of 7 different suture stitches (Vicryl rapide, PDS, Ethilon, Vicryl, Monocryl, Mersilene and Prolene, Johnson \& Johnson MEDICAL GmbH, Germany) on the patient's upper right arm. After $48 \mathrm{~h}$ at all 7 testing sites, erythematous papules up to $3 \mathrm{~mm}$ in diameter were visible (fig. 2c). Histopathological examination of a biopsy specimen from the upper right arm showed a superficial mixed cell infiltrate of neutrophilic, but also some eosinophilic granulocytes. These findings were consistent with the pathergy phenomenon of PG. The patient's past 
medical history revealed no PG-associated comorbidities. Detailed examination failed to detect any systemic complications including inflammatory bowel diseases, hematological disorders or rheumatoid arthritis. Laboratory examination of peripheral blood was unsuspicious, including a negative serology for syphilis. Topical treatment with a triamcinolone acetonide-containing ointment significantly improved healing, and the patient suffered no relapses afterwards (fig. 2b).

\section{Discussion}

The face is not a common location for PG. In one of the largest case series of PG, only $7.8 \%$ appeared on the head or neck [2]. The leg was the most frequent site of PG (77.7\%), followed by the trunk (11.7\%), peristomal area, and the upper extremities (each 8.7\%). PG affecting the lips was only reported twice $[3,4]$. Our first patient suffered from ulceration on the lateral corner of the eye near the left temple. A similar location was recently described by Persing et al. [5] in a 37-year-old woman. This woman developed a PG on the right cheek after re-excision and closure of a biopsy scar, suggesting that pathergy plays a role. Another paper reported on a 44-year-old woman who developed PG on the forehead and the left temporal area without any prior trauma or associated pathologies [6]. In both cases, the diagnosis of superficial granulomatous pyoderma (SGP) was made. SGP is considered to be a chronic, superficial and less aggressive variant of the classic PG [7]. In contrast to classic PG, the lesions have a relatively clean base and vegetative borders, are less inflamed and often indolent.

In both cases, the diagnosis of PG, likely of the SGP type, was made on the basis of clinical and histopathological correlation. Patient 1 also suffered from rheumatoid arthritis, which is frequently associated with PG. In the retrospective review of Binus et al. [2], 34\% of patients with PG suffered from inflammatory bowel disease, $29.1 \%$ from arthritis, and $20.4 \%$ from hematological disorders. In $21.4 \%$ of patients, none of the common comorbidities was found. Tissue pathergy, reported as initiation of new lesions from sites of previous trauma, was documented in $31.1 \%$. Our second patient developed PG after tissue pathergy, namely after surgery of a squamous cell carcinoma on the lower lip. Moreover, a positive pathergy reaction appeared after the intracutaneous insertion of different suture material, on the patient's upper right arm, without any evidence of clinical allergic contact dermatitis. A type IV allergic reaction seemed unlikely. Allergic reactions to suture material are a rare condition $[8,9]$.

The histological findings of PG are not specific. The primary objective in obtaining a biopsy specimen was to exclude the relevant differential diagnoses. In both cases, we ruled out other causes of cutaneous ulcerations such as infections, primary vasculitis, and neoplastic diseases. In combination with the typical clinical appearance, both major criteria of PG defined by Su et al. [1] were fulfilled. Furthermore, the histological findings could be compatible with $\mathrm{PG}$ in both cases. The first patient suffered from a disease strongly associated with PG (and moreover, the lesion resolved under a permanent immunosuppressive treatment with prednisolone and methotrexate) and the history of the second patient was suggestive for pathergy, so that, with 2 major and 2 minor criteria, the diagnosis of PG was confirmed in both patients.

Compared to the average age of PG patients, ours had an advanced age. So far, only a couple of cases demonstrating the appearance of PG in senescent people are reported. Inan et al. [10] described a 90-year-old woman with myelodysplastic syndrome, developing an 
inflammatory skin reaction at the site of the central venous access device insertion. Another case of PG affecting a 92-year-old woman was reported by Topinková et al. [11].

Systemic steroids are by far the most commonly prescribed treatment in patients with PG [2]. Because PG typically responds to moderate doses of systemic corticosteroids, dramatic improvement after initiation of corticosteroid therapy may confirm the diagnosis [1]. A rapid treatment response was also detectable in our first patient. After 7 days of corticosteroid treatment, the wound showed signs of healing. Regrettably, the patient did not turn up at any follow-ups. In analogy, patient 2 showed a rapid improvement of the wound after the application of a topical steroid, and stable wound healing was reached after 4 months. In addition to topical calcineurin inhibitors and intralesional corticosteroids, topical corticosteroids are one of the most common topical treatments in PG [2]. Above all, in localized and limited PG, a topical monotherapy with corticosteroids is a reasonable treatment option, among others because of the avoidance of uncommon adverse effects and the low number of contraindications $[12,13]$. Minor response rates compared to systemic treatment and a higher number of relapses during the course of the disease are the disadvantages of topical treatments [13].

\section{Conclusion}

Our cases indicate that, despite an expected mean age of PG around 50 years, senescent patients can be affected by this inflammatory dermatosis. Moreover, facial PG appears to be more frequent than so far expected. Finally, PG should, after exclusion of relevant differential diagnoses, always be considered in cases of non-healing ulcers, combined with its typical clinical appearance.

\section{Acknowledgement}

The authors wish to thank Markus Karsten for taking and editing the photos.

\section{References}

1 Su WP, Davis MD, Weenig RH, Powell FC, Perry HO: Pyoderma gangrenosum: clinicopathologic correlation and proposed diagnostic criteria. Int J Dermatol 2004;43:790-800.

7 Binus AM, Qureshi AA, Li VW, Winterfield LS: Pyoderma gangrenosum: a retrospective review of patient characteristics, comorbidities and therapy in 103 patients. Br J Dermatol 2011;165:1244-1250.

-3 Poiraud C, Gagey-Caron V, Barbarot S, Durant C, Ayari S, Stalder JF: Cutaneous, mucosal and systemic pyoderma gangrenosum. Ann Dermatol Venereol 2010;137:212-215.

-4 Allen CP, Hull J, Wilkison N, Burge SM: Pediatric pyoderma gangrenosum with splenic and pulmonary involvement. Pediatr Dermatol 2013;30:497-499.

5 Persing SM, Laub D: Superficial granulomatous pyoderma of the face: a case report and review of literature. Eplasty 2012;12; 472-477.

-6 Lachapelle JM, Marot L, Jablonska S: Superficial granulomatous pyoderma gangrenosum of the face, successfully treated by cyclosporine: a long term follow-up. Dermatology 2001;202:155-157.

-7 Hardwick N, Cerio R: Superficial granulomatous pyoderma. A report of two cases. Br J Dermatol 1993;129:718-722.

-8 Kofler L, Radl A, Hoffer Z, Kofler H: Contact allergy to surgical suture material. Allergologie 2002;35:433435.

-9 Sánchez-Morillas L, Reaño Martos M, Rodríguez Mosquera M, Iglesias Cadarso A, Pérez Pimiento A, Domínguez Lázaro AR: Delayed sensitivity to Prolene. Contact Dermatitis 2003;48:338-339.

-10 Inan I, Myers P0, Braun R, Hagen ME, Morel P: Pyoderma gangrenosum after totally implanted central venous access device insertion. World J Surg Oncol 2008;6:31. 
Kratzsch et al.: Facial Pyoderma Gangrenosum in Senescence

11 Topinková E, Klán J, Pelísková D, Dolezelová I: Atypical pyoderma gangrenosum in 92-years-old patient. Cas Lek Cesk 2004;143:481-484.

12 Le Cleach L, Moguelet P, Perrin P, Chosidow O: Is topical monotherapy effective for localized pyoderma gangrenosum? Arch Dermatol 2011;147:101-103.

13 Wenzel J, Gerdsen R, Phillipp-Dormston W, Bieber T, Uerlich M: Topical treatment of pyoderma gangrenosum. Dermatology 2002;205:221-223.

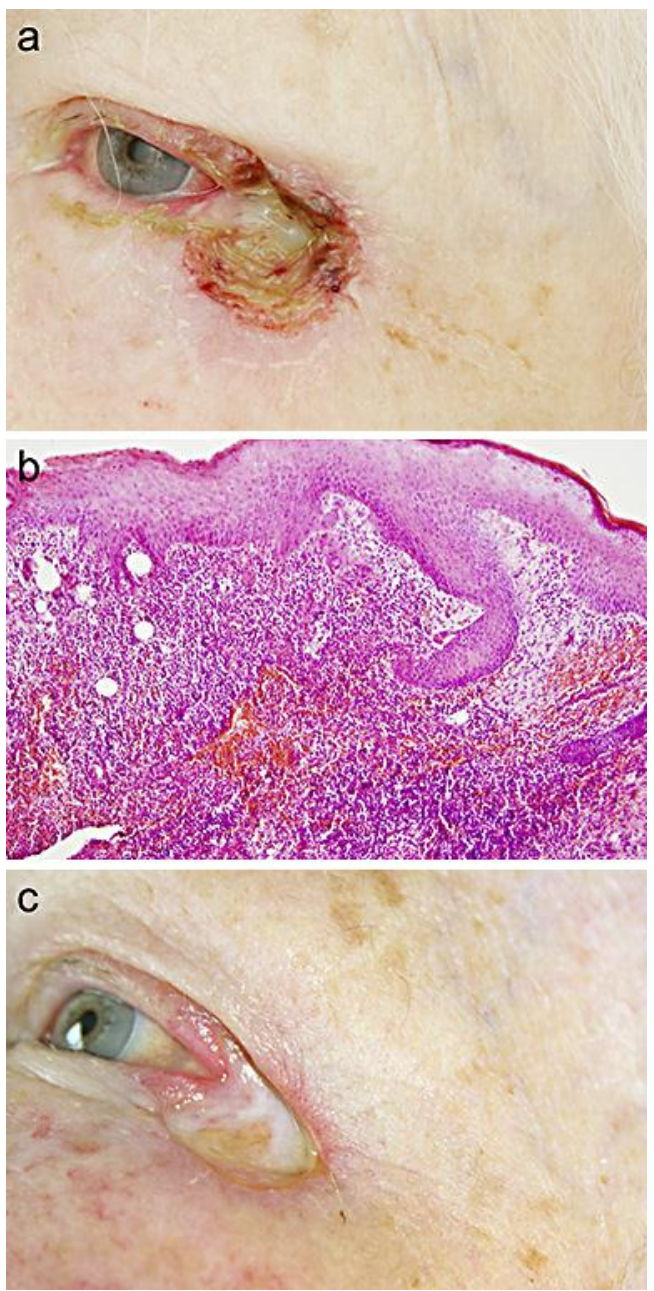

Fig. 1. Clinical and histopathological features of case 1 a An $1.5 \times 1.5 \mathrm{~cm}$ ulcer on the lateral corner of the left eye with a necrotic and fibrinoid surface. b Histopathological examination of a biopsy specimen showed a dense, mixed-cell, dermal infiltrate with numerous neutrophilic granulocytes and some macrophages. c Less necrotic and clean base as well as less inflammatory borders after 2 weeks of systemic corticosteroid therapy. 

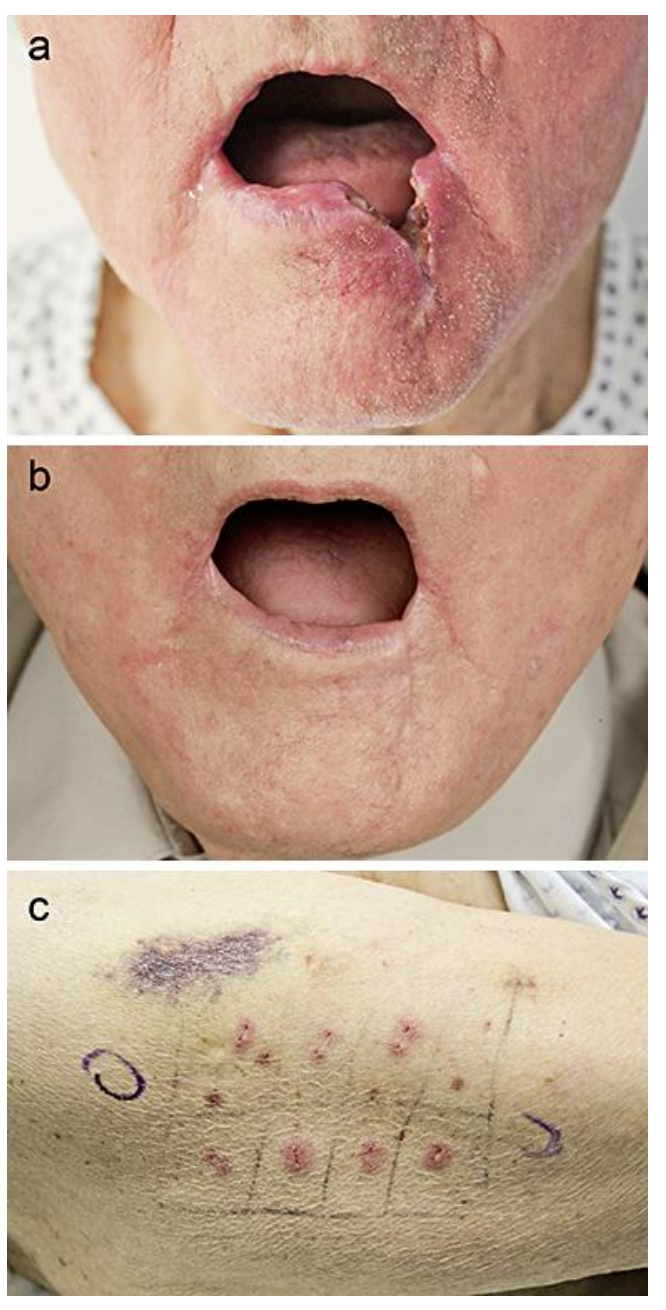

Fig. 2. Clinical and histopathological features of case 2. a Painful ulcer with violaceous, undermined borders on the left part of the lower lip. b Stable remission after 4 months of treatment. c After 48 h, all 7 testing sites showed erythematous papules up to $3 \mathrm{~mm}$ in diameter. 